\title{
Potential Clinical Usefulness of the Polymerase Chain Reaction Test to Detect Pathogens Causing Sepsis
}

\author{
${ }^{1}$ Department of Trauma, Critical Care Medicine and Burn Center, Social Insurance Chukyo Hospital, Nagoya Japan \\ ${ }^{2}$ Department of Traumatology and Acute Critical Medicine, Osaka University Graduate School of Medicine, Japan \\ ${ }^{3}$ Department of Emergency Room Medicine, Kinki University, School of Medicine, Japan \\ ${ }^{4}$ Laboratory for Clinical Investigation, Osaka University Graduate School of Medicine, Japan \\ ${ }^{5}$ Hoshigaoka Koseinenkin Hospital, Japan
}

Asako Matsushima ${ }^{1 *}$, Osamu Tasaki ${ }^{2}$, Takeshi Shimazu ${ }^{3}$, Seishi Asari ${ }^{4}$, Keigo Kimura ${ }^{4}$, Tomomi Sakata ${ }^{4}$, Hisashi Sugimoto ${ }^{5}$

\begin{abstract}
Objective: A real-time polymerase chain reaction (PCR) test is expected for early and precise detection of pathogens in blood. In this study, we compared the ability of the PCR test and blood culture to detect pathogens in the blood of patients with sepsis.

Methods: Patients who were diagnosed as or suspected of having sepsis were included in this prospective observational study. A whole blood sample for PCR test was obtained serially simultaneously with the blood culture sample, and the results were compared.

Results: We obtained 93 samples from 26 patients; 69 samples were obtained during the septic condition, and 24 samples were from the non-septic condition. Origins of sepsis were pneumonia in 9 patients, necrotizing fasciitis in 5 patients, and other causes in 12 patients. In the septic condition, rates of positive results were $29.0 \%$ for the PCR test and $23.2 \%$ for blood culture. Sample contamination occurred in 1 PCR test sample and 5 blood culture samples. In positive PCR samples, 16 of 20 samples were obtained during sepsis after beginning administration of broadspectrum antibiotics, whereas 5 of 12 samples, except for contaminated samples, were positive in blood culture.
\end{abstract}

Conclusion: In sepsis, the PCR test detected more bacteria than did blood culture even after administration of empirical antibiotics, which might contribute to precise diagnosis of the bacteremic cause of sepsis.

Keywords: Real-time polymerase chain reaction test; Sepsis; Blood culture

\section{Introduction}

Sepsis is a leading cause of death worldwide. Every year, more than 18 million people suffer from sepsis, and 1400 people die of sepsis every day $[1,2]$. The Surviving Sepsis Campaign guidelines declared in 2004 pointed out the extremely high mortality rate of severe sepsis and septic shock and therefore emphasized the early recognition of sepsis and the beginning of broad-spectrum antibiotic treatment as early as possible to prevent deterioration of the patient's condition [3]. This "early empirical therapy" is necessary until the origins of sepsis become clear; however, it would be better to know the precise target of therapy before administration of antibiotics so that the target microorganism is not missed and growth of antibiotic-resistant bacteria can be avoided.

To detect the origin of sepsis, Gram staining and culturing of samples from the infection site, as well as blood culture (BC), are the universal methods. Gram staining has been reevaluated recently as a quick, easy, and inexpensive way to detect the target microorganisms in infections [4,5]. However, because of the lower number of microorganisms in the blood in comparison with infection site, they are difficult to detect with Gram staining. In contrast, BC is the gold standard for detecting bacteremia and fungemia, but it takes at least several days to grow the microorganisms, and false negatives due to previous antibiotic use or the presence of fastidious intracellular pathogens are well known limitations of BC.

The polymerase chain reaction (PCR) test was developed as an attractive method to detect pathogens in blood within 5 hours, which is expected to aid in the early and precise diagnosis of the origins of sepsis. To date, significantly higher positive results from the PCR test than BC have been shown in multiple trials targeting sepsis $[6,7,8]$; however, antibiotic resistance determinants or interpretation of false-positive PCR tests are the major limitations preventing wide clinical use of this method. In their review in 2011, Pletz et al. [9] discussed the clinical advantages and cost effectiveness of PCR testing and stated that the PCR test should focus on those pathogens or resistance determinants that are not covered by guideline-recommended treatment regimens and that have been identified as the major cause of inappropriate treatment according to current studies: Candida ssp., Aspergillus spp., methicillin-resistant Staphylococcus aureus (MRSA), vancomycinresistant enterococci, and extended-spectrum beta-lactamase and carbapenemase-positive Gram-negative microorganisms. However, in regard to the positive results of PCR tests, Bloos et al. [10] reported in 2010 that patients with positive PCR tests at enrollment had higher organ dysfunction scores and a trend toward higher mortality in comparison with those with negative PCR results. They concluded that PCR test results correlated with disease severity even if the $\mathrm{BC}$ remained negative and suggested that presence of microbial DNA in the bloodstream is a significant event.

*Corresponding author: Asako Matsushima, Department of Trauma, Critical Care Medicine and Burn Center, Japan, Tel: 81-52-691-7151; Fax: 81-52-6925220; E-mail: asakouab@yahoo.co.jp

Received January 19, 2012; Accepted March 01, 2012; Published March 09, 2012

Citation: Matsushima A, Tasaki O, Shimazu T, Asari S, Kimura K et al. (2012) Potential Clinical Usefulness of the Polymerase Chain Reaction Test to Detect Pathogens Causing Sepsis. J Medical Microbiol Diagnosis 1:106. doi:10.4172/21610703.1000106

Copyright: (C) 2012 Matsushima A, et al. This is an open-access article distributed under the terms of the Creative Commons Attribution License, which permits unrestricted use, distribution, and reproduction in any medium, provided the original author and source are credited. 
In the present study, we hypothesized that positive PCR test results would help to diagnose pathogens causing sepsis even if broadspectrum antibiotics had already been administered before blood sampling. We therefore obtained serial blood samples from septic patients to compare the results of PCR testing and BC during use of broad-spectrum antibiotics.

\section{Patients and Methods}

This prospective observational study was performed from September 2007 to April 2008 in the medical and surgical intensive care unit in the emergency department of Osaka University Graduate School of Medicine, Osaka, Japan. Patients who were admitted to the intensive care unit and who were either diagnosed as having or were suspected of having sepsis were included. Patients who visited the emergency room only were excluded from this study. We diagnosed sepsis according to the definitions of the American College of Chest Physicians (ACCP) and the Society of Critical Care Medicine (SCCM) [11]. Samples of 1.5 $\mathrm{ml}$ of whole blood were collected from each patient for the PCR test together with a $10-\mathrm{ml}$ whole blood sample for BC $(5 \mathrm{ml}$ for aerobic culture and $5 \mathrm{ml}$ for anaerobic culture) via venipuncture, when BC was needed for diagnosis of sepsis or evaluation of antibiotic treatment. Blood culture was performed according to the standard technique in our clinical microbiologic laboratory with the BacT/ALERT 3D system (BioMerieux; Hazelwood, MO, USA). Whole blood for PCR testing was collected into an ethylenediaminetetraacetic acid (EDTA) tube, and DNA extraction and amplification were performed separately to eliminate contamination. DNA extraction and amplification were performed according to the instructions of the SeptiFast test (Roche Diagnostics; Mannheim, Germany), and the details of the method are described elsewhere [12]. Briefly, microbe DNA from each patient's sample was extracted using the SeptiFast Lys and Prep Kit M Grade (Roche Diagnostics). DNA amplification was performed on the LightCycler 2.0 system (Roche Diagnostics) using a bacteria and fungi identification macro included in the Light-Cycler software V4.05. The SeptiFast mecA test to differentiate MRSA was performed after $S$. aureus was detected, except when coagulase-negative Staphylococcus (CNS) was found, because CNS also carries the mecA gene [13]. The analytical sensitivity of the PCR test is 30 colony-forming units (CFU)/ $\mathrm{mL}$ for organisms listed in Table 1, except for CNS, Streptococcus species, and Candida glabrata, for which the minimum sensitivity is $100 \mathrm{CFU} / \mathrm{mL}$ [14].

The medical or surgical staff treating the patients were blinded to the results of the PCR test. Tree medical staff members including an infection disease specialist reviewed the patient's clinical records such as vital signs, laboratory results, antibiotics used, and the treatment course, and categorized each sampling point as occurring during the septic condition (sepsis) or during the non-septic condition (nonsepsis). Presence of CNS was regarded as contamination unless it was detected from 2 sets of $\mathrm{BC}$ bottles or detected from $\mathrm{BC}$ more than 2 times in a row or detected from other culture specimens obtained from aseptic sites (i.e., urine, catheter, wound, or body cavity). Infection was defined according to the International Sepsis Forum consensus [15]. The international definitions of the SCCM, ACCP, the European Society of Intensive Care Medicine (ESICM), the American Thoracic Society (ATS), and the Surgical Infection Society (SIS) were used as references to differentiate sepsis from non-sepsis for each sample [16].

This study was approved by the institutional review board of the Osaka University Graduate School of Medicine.

\section{Results}

Twenty-six patients were enrolled in this study. The patient population consisted of 16 men and 10 women, median age 66 (range 39-91) years. In 20 of the 26 patients, samples for both BC and PCR test were obtained serially during sepsis or to evaluate antibiotic effect, and then 93 samples from the 26 patients were collected (average 3.6 \pm 2.9 samples for each patient). Sixty-nine samples were obtained during sepsis, and 24 samples were during non-sepsis. The origins of sepsis were pneumonia in 9 patients, necrotizing fasciitis in 5 patients, peritonitis in 3 patients, and other origins in 9 patients (Table 2).

The percentage of positive detections by PCR test and BC in samples obtained during sepsis and non-sepsis are shown in Figure 1. In sepsis, the PCR test detected pathogens in 20 of 69 (29.0\%) samples, whereas BC detected them in 12 of $69(17.4 \%)$ samples (8 samples were listed on the PCR test menu, and 4 samples were not listed on the PCR test menu). Neither PCR test nor BC detected pathogens in 24 non-sepsis samples. Contamination was determined in the $\mathrm{BC}$ in 4 samples from sepsis and 1 sample from non-sepsis, whereas no sample from sepsis and 1 sample from non-sepsis were determined to be contaminated in the PCR test. In the results from sepsis, 16 of 20 positive PCR samples, in which pathogens were all diagnosed as origins of sepsis, were obtained after the administration of broadspectrum antibiotics. However, 7 positive BC samples were obtained before administration of antibiotics, and 5 samples, except for those determined to be contaminated, were obtained after the administration of effective antibiotics.

Comparison of PCR test and BC in cases with a positive PCR test and negative $\mathrm{BC}$ during antibiotics administration are shown in Figure 2. The details of each case are described in the figure caption. All blood samples in these cases were obtained during sepsis. In each

\begin{tabular}{|l|l|l|}
\hline Gram positive & Gram negative & Fungi \\
\hline Staphylococcus aureus & Escherichia coli & Candida albicans \\
\hline CNS & $\begin{array}{l}\text { Klebsiella (pneumoniae/ } \\
\text { oxyt.) }\end{array}$ & Candida tropicalis \\
\hline $\begin{array}{l}\text { Streptococcus } \\
\text { pneumoniae }\end{array}$ & Serratia marcescens & Candida parapsilosis \\
\hline Streptococcus spp. & $\begin{array}{l}\text { Enterobacter (cloacae/ } \\
\text { aerog.) }\end{array}$ & Candida krusei \\
\hline Enterococcus faecium & Proteus mirabilis & Candida glabrata \\
\hline Enterococcus faecalis & Pseudomonas aeruginosa & Aspergillus (fumigatus) \\
\hline MRSA (mecA gene) & Acinetobacter baumannii & \\
\hline & $\begin{array}{l}\text { Stenotrophomonas } \\
\text { maltophilia }\end{array}$ & \\
\hline
\end{tabular}

CNS, coagulase-negative Staphylococcus aureus; MRSA, methicillin-resistant Staphylococcus aureus.

Table 1: Microorganisms Detected by the Polymerase Chain Reaction Test.

\begin{tabular}{|l|c|}
\hline Infection & Number of patients \\
\hline Pneumonia & 9 \\
\hline Necrotizing fascitis & 5 \\
\hline Peritonitis & 3 \\
\hline Retroperitonitis & 2 \\
\hline Meningitis & 2 \\
\hline Phlebitis & 2 \\
\hline Cholangitis & 1 \\
\hline Mediastinitis & 1 \\
\hline Sinusitis & 1 \\
\hline Total & 26 \\
\hline
\end{tabular}

Table 2: Number of Patients According to the Origin of Sepsis. 


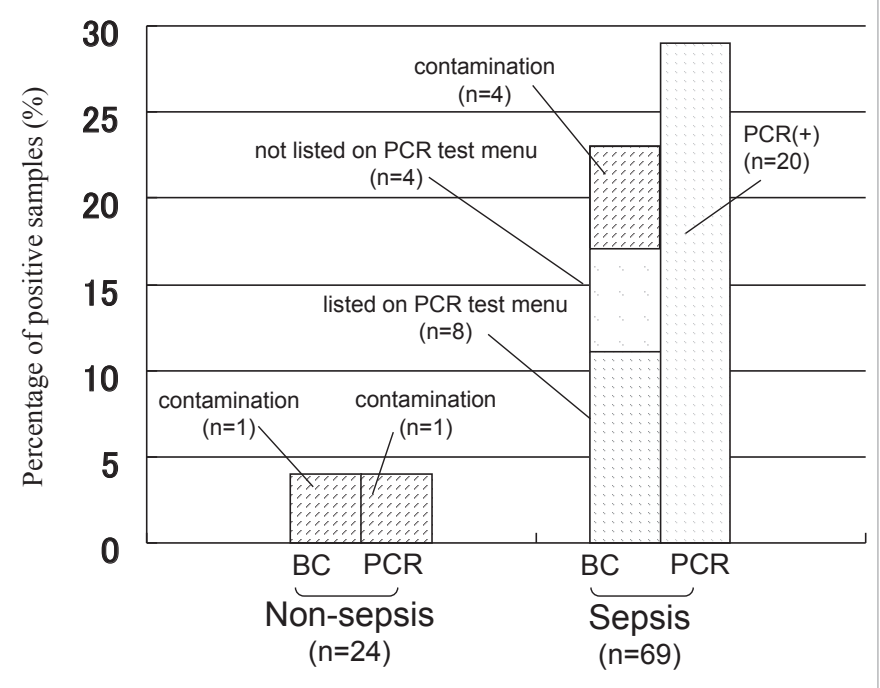

Figure 1: The percentage of positive polymerase chain reaction $(\mathrm{PCR})$ test and blood culture $(\mathrm{BC})$ samples in sepsis and non-sepsis. PCR, polymerase chain reaction; $\mathrm{BC}$, blood culture.

case, the PCR test showed a positive result even after administration of antibiotics, whereas the $\mathrm{BC}$ was negative, and the microorganisms identified in these positive PCR results were all confirmed as the origins of sepsis by other samples obtained from the infection site. Because of the protocol of this study, neither the medical or surgical staffs were informed of the PCR results, nor were the results reflected in the patients' treatment.

The numbers of PCR tests and/or BCs positive for pathogens are summarized in Table 3. BC detected CNS in 5 samples that were eventually determined to be contaminated, whereas the PCR test did not detect any CNS. The PCR test detected S. aureus in one sample, which was determined to be contamination after a review of the clinical records. All positive results except for contamination were confirmed as the cause of sepsis. The results from the blood sample were consistent with other samples obtained from the infection site including those in cases of meningitis, cholangitis, mediastinitis, and retroperitonitis, in which the samples were obtained by surgical or radiological procedures.

Five samples were confirmed as MRSA infection by BC or other culture techniques. The PCR test detected S. aureus in these 5 samples; however, the mecA gene was detected in only 1 sample.

\section{Discussion}

Early diagnosis and proper use of antibiotics is essential to treat sepsis. Empirical broad-spectrum antibiotics were recommended by the Surviving Sepsis Campaign guidelines; however, precise diagnosis of the target microorganism and early determination of definitive antibiotic therapy are still challenges to be overcome. In the present study, we have shown some value in the new method using realtime PCR to diagnose the cause of sepsis. This method is designed to detect bacteria and fungi in whole blood by the amplification of their nucleic acids within 5 hours, which would make it possible to obtain information on bacteremia and fungemia quite earlier than that which could be obtained from BC. The higher sensitivity of this method compared with $\mathrm{BC}$ is reported in many recent studies $[6,7,8]$; however, the interpretation of positive PCR test results was not consistent, especially when $\mathrm{BC}$ results were negative [17]. In their multicenter trial to compare $\mathrm{PCR}$ test and $\mathrm{BC}$, Bloos et al. [10] reported $34.7 \%$ positive results with the PCR test in comparison with $16.5 \%$ positive results for BC in severe sepsis. Their study showed higher organ dysfunction scores and a trend toward higher mortality in patients with positive PCR results, and they concluded that positive PCR results (presence of microbial DNA in the bloodstream) were a significant event even if the $\mathrm{BC}$ remained negative. We also showed a higher rate of positive results for the PCR test versus BC in sepsis (20/69 [29.0\%] vs. 12/69 [17.4\%], excluding contamination), and all positive PCR samples except 1 with contamination were confirmed as detecting the bacteria causing the sepsis. Of the 20 positive PCR samples in sepsis, 16 were collected after broad-spectrum antibiotic administration had started. These included cases of meningitis, cholangitis, mediastinitis, and retroperitonitis, in which it is sometimes difficult to obtain samples from the site of infection. In such cases, broad-spectrum antibiotics are administered immediately after diagnosis on the basis of clinical findings and/or radiographic images along with sampling for BC. The surgical approaches of resection or drainage would be undertaken, but the original microorganism that caused the sepsis is not always detected in the presence of broad-spectrum antibiotics. As shown in Figure 2, the PCR test was positive in sepsis in the presence of broadspectrum antibiotics even if the $\mathrm{BC}$ was negative. The microorganisms identified in the positive PCR results were confirmed as the origins of sepsis later from samples obtained at the site of infection. Although we did not inform the medical or surgical staff of these results because of the requirements of the study protocol, it is possible that if the PCR test results can be obtained within 5 hours, the strategy to treat sepsis or antibiotics used could be changed earlier in the treatment course. Although the sample size in the present study is small, we believe our results showed some benefit of the PCR test in clinical use, especially when broad-spectrum antibiotics are being used.

Another concern regarding positive PCR results is contamination. In this study, only one sample was determined to be contaminated by $S$. aureus in PCR testing, whereas 5 samples were contaminated by CNS according to the $\mathrm{BC}$ results. One reason for lower CNS detection is the higher threshold of analytical sensitivity of CNS than for other pathogenic microorganisms in the PCR test. CNS is usually considered as contamination, but Casalta et al. [18] pointed out that this higher threshold of the PCR test for CNS risks low-grade bloodstream infections, such as endocarditis, being overlooked during antibiotic treatment. To distinguish true bacteremia from contamination, Rowther et al. [19] reported the usefulness of PCR testing combined with serum procalcitonin. They reported that positive PCR results with elevated procalcitonin levels suggested true sepsis, and negative PCR results with reduced procalcitonin levels suggested non-sepsis. Additional examination and careful evaluation is needed to decide between contamination and bacteremia in these septic patients.

One limitation of the present PCR test was its inability to detect microorganisms that were not listed on the test menu. There were 4 samples in which bacteria detected by BC were not on the PCR test menu but were the cause of sepsis. Citrobacter, which is now increasingly a cause of sepsis in compromised hosts [20,21], is one of them. The other 3 samples included anaerobic bacteria, Bacteroides species, and Fusobacterium species, which have been highlighted recently as causes of severe sepsis [22]. In the case of anaerobes in particular, they take more time to grow in $\mathrm{BC}$ due to their specialized culture requirements, and it is therefore desirable to include these bacteria in the PCR test menu in the future. The second limitation of the PCR test was the lower sensitivity of the mecA gene to distinguish MRSA from methicillinsensitive $S$. aureus. Although we reexamined these samples, the mecA gene was detected in only one sample. Information about multidrug 
Citation: Matsushima A, Tasaki O, Shimazu T, Asari S, Kimura K et al. (2012) Potential Clinical Usefulness of the Polymerase Chain Reaction Test to Detect Pathogens Causing Sepsis. J Medical Microbiol Diagnosis 1:106. doi:10.4172/2161-0703.1000106

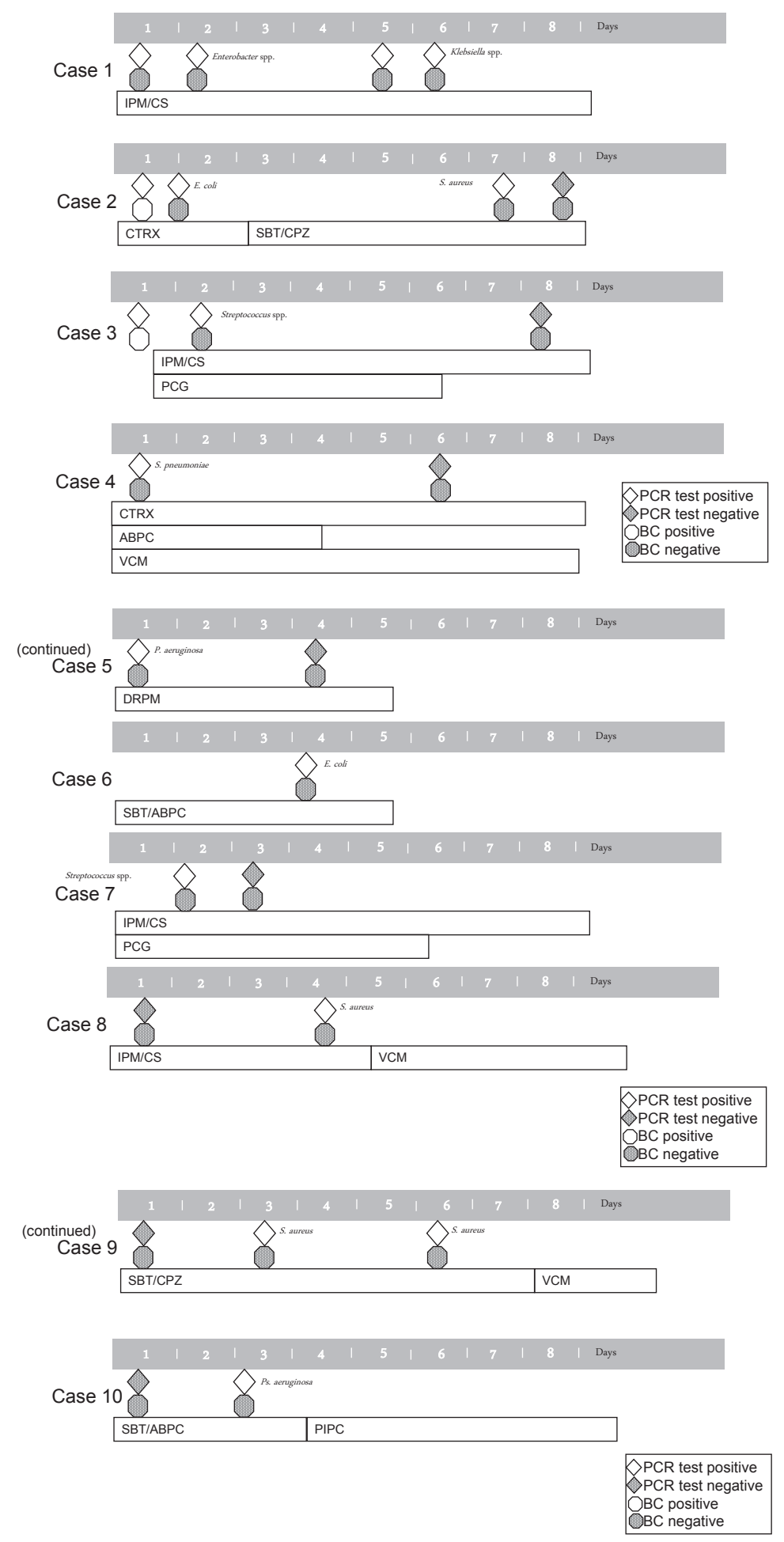

Figure 2: Comparison of polymerase chain reaction (PCR) test and blood culture $(B C)$ in cases with positive PCR test in which antibiotics were administered. Case 1: A 45-year-old man with retroperitonitis due to pancreatitis. Enterobacter spp. and Klebsiella spp. were detected in samples obtained by surgical procedure. Case 2: A 63-year-old woman with cholecystitis. Escherichia coli was detected in purulent bile. Enteritis due to MRSA became a complication after day 7. Case 3: A 63-year-old man with mediastinitis due to pharyngitis. Streptococcus spp. was detected in samples obtained by surgical procedure. Case 4: A 67-year-old woman with meningitis. Streptococcus pneumoniae was detected in cerebrospinal fluid. Case 5: A 69-year-old man with retroperitonitis due to pancreatitis. Pseudomonas aeruginosa was detected in samples obtained by surgical procedure. Case 6: An 89-year-old woman with urinary tract infection complicated by pneumonia. E. coli was detected in a urinary sample. Case 7: A 61-year-old woman with necrotizing fascitis due to pharyngitis. Streptococcus spp. was detected in samples obtained by surgical procedure. Case 8: A 53-year-old man with necrotizing fascitis of the bilateral lower limbs. Staphylococcus aureus was detected in samples obtained by surgical procedure. Case 9: A 58-year-old man with abdominal abscess due to traumatic peritonitis. S. aureus was detected in samples obtained by surgical procedure. Case 10: A 77-year-old man with pneumonia. $P$. aeruginosa was detected in samples of tracheal aspirate. 


\begin{tabular}{|l|c|c|c|}
\hline & $\begin{array}{c}\text { PCR(+) BC(+) } \\
\text { (n) }\end{array}$ & $\begin{array}{c}\text { PCR(+) } \\
\mathbf{B C}(-) \\
(\mathbf{n})\end{array}$ & $\begin{array}{c}\text { BC(+) PCR(-) } \\
\text { (n) }\end{array}$ \\
\hline Pathogens on the PCR test menu & \multicolumn{2}{|l|}{} \\
\hline Coagulase-negative Staphylococcus & 0 & 0 & 5 \\
\hline Staphylococcus aureus & 2 & 5 & 2 \\
\hline Streptococcus species & 1 & 2 & 0 \\
\hline Streptococcus pneumoniae & 0 & 1 & 0 \\
\hline Escherichia coli & 1 & 2 & 0 \\
\hline Enterobacter aerogenes/cloacae & 0 & 2 & 0 \\
\hline Klebsiella pneumoniae/oxytoca & 0 & 2 & 0 \\
\hline Pseudomonas aeruginosa & 1 & 2 & 1 \\
\hline Pathogens not on the PCR test menu & & & \\
\hline Citrobacter species & 0 & 0 & 1 \\
\hline Bacteroides species & 0 & 0 & 2 \\
\hline Fusobacterium species & 0 & 0 & 1 \\
\hline
\end{tabular}

$\mathrm{PCR}$, polymerase chain reaction; $\mathrm{BC}$, blood culture.

Table 3: Summary of PCR Test and BC Results According to Pathogen.

resistance is quite important when choosing antibiotics. As Pletz et al. [9] stated, PCR test is expected to focus on resistance determinants for more cost effective and to meet current emerging clinical needs, so further improvement in the detection of the mecA gene or other multidrug resistance genes of Gram-negative bacteria is also desired for the PCR test in the future.

\section{Conclusion}

In conclusion, the PCR test detected more bacteria during sepsis, even after administration of empirical antibiotics, than did BC. The PCR test might contribute to precise diagnosis of bacteremic cause of sepsis.

\section{Acknowledgements}

We thank Dr. Katsunori Yanagihara, Dr. Yuko Kitagawa, Dr. Yoshinobu Sumiyama, Dr. Masaki Kitajima, and Roche Diagnostics for their help in this study.

\section{References}

1. Angus DC, Linde-Zwirble WT, Lidicker J, Clermont G, Carcillo J, et al. (2001) Epidemiology of severe sepsis in the United States: analysis of incidence, outcome, and associated costs of care. Crit Care Med 29: 1303-1310.

2. Bone RC, Balk RA, Cerra FB, Dellinger RP, Fein AM, et al. (1992) Definitions for sepsis and organ failure and guidelines for the use of innovative therapies in sepsis. The ACCP/SCCM Consensus Conference Committee. Chest 101: 1644-1655.

3. Dellinger RP, Levy MM, Carlet JM, Bion J, Parker MM, et al. (2008) Surviving Sepsis Campaign: International guidelines for management of severe sepsis and septic shock. Crit Care Med 36: 296-327.

4. Matsushima A, Tasaki O, Shimizu K, Tomono K, Ogura H, et al. (2008) Preemptive antibiotic treatment based on gram staining reduced the incidence of ARDS in mechanically ventilated patients. J Trauma 65: 309-315.

5. Aygun G, Yasar H, Yilmaz M, Karasahin K, Dikmen Y, et al. (2006) The value of Gram staining of catheter segments for rapid detection of peripheral venous catheter infections. Diagn Microbiol Infect Dis 54: 165-167.

6. Lehmann LE, Alvarez J, Hunfeld KP, Goglio A, Kost GJ, et al. (2009) Potential clinical utility of polymerase chain reaction in microbiological testing for sepsis. Crit Care Med 37: 3085-3090

7. Westh H, Lisby G, Breysse F, Boddinghaus B, Chomarat M, et al. (2009) Multiplex real-time PCR and blood culture for identification of bloodstream pathogens in patients with suspected sepsis. Clin Microbiol Infect 15: 544-551.

8. Lucignano B, Ranno S, Liesenfeld O, Pizzorno B, Putignani L, et al. (2011) Multiplex PCR allows rapid and accurate diagnosis of bloodstream infections in newborns and children with suspected sepsis. J Clin Microbiol 49: 2252-2258.

9. Pletz MW, Wellinghausen N, Welte T (2011) Will polymerase chain reaction

(PCR)-based diagnosis improve outcome in septic patients? A clinical view Intensive Care Med 37: 1069-1076.

10. Bloos F, Hinder F, Becker K, Sachse S, Mekontso Dessap A, et al. (2010) A multicenter trial to compare blood culture with polymerase chain reaction in severe human sepsis Intensive Care Med 36: 241-247.

11. No authors listed (1992) American College of Chest Physicians/Society of Critical Care Medicine Consensus Conference: definitions for sepsis and organ failure and guidelines for the use of innovative therapies in sepsis. Crit Care Med 20: 864-874.

12. Louie RF, Tang Z, Albertson TE, Cohen S, Tran NK, et al. (2008) Multiplex polymerase chain reaction detection enhancement of bacteremia and fungemia. Crit Care Med 36: 1487-1492.

13. Chaieb K, Touati A, Salah AM, Hassen AB, Mahdouani K, et al. (2006) DNA fingerprinting of a multi-resistant coagulase-negative staphylococci isolated from biomaterials in dialysis services. Arch Med Res 37: 953-960.

14. Light Cycler Septi Fast Test. Package Insert. Roche Diagnostics GmbH. 2006

15. Calandra T, Cohen J (2005) The international sepsis forum consensus conference on definitions of infection in the intensive care unit. Crit Care Med 33: $1538-1548$.

16. Levy MM, Fink MP, Marshall JC, Abraham E, Angus D, et al. (2003) 2001 SCCM/ESICM/ACCP/ATS/SIS International Sepsis Definitions Conference. Intensive Care Med 29: 530-538.

17. Schrenzel J (2011) PCR for the diagnosis of sepsis: hope or hype? Crit Care 15: 111.

18. Casalta JP, Gouriet F, Roux V, Thuny F, Habib G, et al. (2008) Evaluation of the LightCycler ${ }^{\circledR}$ SeptiFast test in the rapid etiologic diagnostic of infectious endocarditis. Eur J Clin Microbiol Infect Dis 28: 569-573.

19. Rowther FB, Rodrigues CS, Deshmukh MS, Kapadia FN, Hegde A, et al. (2009) Prospective comparison of eubacterial polymerase chain reaction (PCR) and procalcitonin with blood culture for diagnosing septicemia in ICU patients. J Clin Microbiol 47: 2964-2969.

20. Miyamoto K, Hiramatsu M, Ashikawa T, et al. (2009) A case of necrotizing softtissue infection caused by Citrobacter koseri. JJAAM 20: 37-41.

21. Mohanty S, Singhal R, Sood S, Dhawan B, Kapil A, et al. (2007) Citrobacter infections in a tertiary care hospital in Northern India. J Infect 54: 58-64.

22. Lassmann B, Gustafson DR, Wood CM, Rosenblatt JE (2007) Reemergence of anaerobic bacteremia. Clin Infect Dis 44: 895-900. 